\title{
Relationship between Masseter Muscle Thickness and Skeletal Muscle Mass in Elderly Persons Requiring Nursing Care in North East Japan
}

\author{
Kento Umeki, ${ }^{1}$ Yutaka Watanabe, ${ }^{2,3}$ and Hirohiko Hirano ${ }^{2,4}$ \\ ${ }^{1}$ Nihon University Graduate School of Dentistry at Matsudo, Removable Prosthodontics, Matsudo, Chiba 271-8587, Japan \\ ${ }^{2}$ Research Team for Promoting Independence of the Elderly, Tokyo Metropolitan Institute of Gerontology, Itabashi, Tokyo \\ 173-0015, Japan \\ Departments of ${ }^{3}$ Preventive and Public Oral Health, ${ }^{4}$ Removable Prosthodontics, Nihon University School of Dentistry at \\ Matsudo, Chiba 271-8587, Japan
}

\section{Article History}

Received 10 December 2016

Accepted 11 January 2017
Keywords :

elderly person requiring nursing

care, skeletal muscle mass,

masseter muscle thickness,

sarcopenia, masticatory function

\begin{abstract}
Maintenance and improvement of masticatory function in nursing care elderly persons (NC) is an important issue, and it is speculated that sarcopenia is related to declining masticatory function. The decrease in skeletal muscle index (SMI), a major diagnostic criterion for sarcopenia, has been reported to be associated with swallowing function in NC. However, the relationship between SMI and masticatory function is unknown. Therefore, we investigated the relationship between masseter muscle thickness (MMT) and SMI, with the aim of examining the specific relationship between decreased masticatory function and sarcopenia in NC. MMT and SMI were measured by ultrasonography and bioelectrical impedance analysis in 275 NC participants in Omori Town, Yokote City, Akita Prefecture in the Tohoku region in Japan. Cognitive functions measured from all participants using questionnaire. Participants were classified into low-MMT or high-MMT group based on the median of each of MMT, and SMI and related items in each gender. In addition, to examine the factors related to MMT, logistic regression analysis was conducted by entering age, sex, SMI, nutrition status, severity of dementia, and other items as explanatory variables and MMT as objective variable. SMI in high-MMT group were significantly higher than low-MMT group (high-MMT: $4.8 \pm 1.4 \mathrm{~kg} / \mathrm{m}^{2}$, low-MMT: $4.4 \pm 1.4 \mathrm{~kg} / \mathrm{m}^{2}, P=0.010$ ). Furthermore, logistic regression analysis indicated that SMI were significantly associated with a MMT (Odds Ratio=0 .83, 95\% Confidence Interval $=0.69-0.99, P=0.049$ ). Our result suggested that the mass of the masseter muscles decreased with NC due to sarcopenia, possibly contributing to a decrease in masticatory function.
\end{abstract}

\section{Introduction}

In Japan where the aging population is increasing annually, maintenance and amelioration of masticatory function in elderly persons are extremely important issues for maintaining not only nutritional status but also quality of life (QOL) through the enjoyment of eating. A decrease in various physical functions is seen in elderly persons requiring nursing care $(\mathrm{NC})$, and masticatory function is no exception. Decreased masticatory function in these elderly persons is a critical issue that is linked with worsening of

Correspondence to :

Yutaka Watanabe

E-mail : ywata@tmig.or.jp
QOL, nutritional status, etc. (1). Especially, it is said that there is a decrease of oral function (Oral-frail) is contributed by "Frail" in elderly persons in recent years (2).

Strategies for ameliorating masticatory function in elderly persons include treatment of caries and periodontal disease in remaining teeth and prosthetic replacement of missing teeth. In recent years, several studies have reported that the decreased function of masticatory factors other than the teeth (e.g., masticatory muscles and the tongue) is responsible for the difficulty in mastication (3). We believed that sarcopenia might be a background factor for the decreased function in masticatory muscles and the tongue. Sarcopenia is primarily characterized by generalized muscle weakening 
in elderly persons (4) and has been reported to be a risk factor for increased mortality of elderly patients in the acute care ward (5). It has also been mentioned that poor nutritional status is a cause of sarcopenia (4), and maintenance of masticatory function may prevent sarcopenia through the maintenance of nutritional status (6). The relationship between masticatory function and sarcopenia in healthy elderly persons has been previously reported (6); however, to our knowledge, there are no studies that have examined the relationship between masticatory function and sarcopenia in NC. NC are different from healthy elderly persons, as they have decreased cognitive function and systemic disease, and it is unclear if a similar relationship exists in healthy elderly persons. Furthermore, early discovery and prevention of decreased masticatory function are extremely important because decreased masticatory function leads to disturbance of bolus formation and the risk of difficulty in swallowing is higher compared with healthy elderly persons. The swallowing function, which is one of oral function items, and skeletal muscle mass has evidence of significant relationship (7). However, the relations between other oral functions (e.g., mastication) and skeletal muscle mass, remain unclear. The principal aims of the present study were to elucidate the relationship between masseter muscle thickness (MMT), which is the muscle that influences masticatory function the most, and skeletal muscle index (SMI), a diagnostic criterion of sarcopenia, and we also examined the specific relationship between decreased masticatory function and sarcopenia.

\section{Materials and Methods}

This study was conducted as part of a survey on NC conducted by Tokyo Metropolitan Institute of Gerontology (TMIG). This project was entitled "Study on Improvement of NC's Oral Function and Oral Health Condition and Improvement of Dietary Life", and supported by a Health and Labor Sciences Research Grant(H25-Choju-Ippan-005).

\section{Participants}

The target population was $399 \mathrm{NC}$, aged 65 to 98 years old, who lives in Omori Town, Yokote City, Akita Prefecture in the Tohoku region of Japan. The population aging rate in this area is $33.1 \%$ as of 2014, and it is higher than national average in Japan. The investigation was complete enumeration of all of the NC who live in this area. Participants were resident of visitors at disability ward, medical ward, health center for the elderly, special nursing home, dementia group home, and day care facility at Omori public hospital, Akita. Among 275 persons ( 60 men and 215 women; mean age, 85.6 \pm 6.5 years) who had agreed to the study participated, analysis were carried out with no missing value in measurement items. The investigation was conducted in February 2014. The reasons for excluding 124 participants are as follows, (1) Pacemaker user, (2) Persons with contracture or loss of limbs, and (3) Residents unable to investigate by entering the facility due to infectious.

\section{Investigation parameters}

For each item, we administered a preliminary survey to the primary caregivers and performed preliminary training of the dentists and dental hygienists who performed the measurements using the methods described below. The selection of items other than the primary investigation items in present study was performed based on previous studies of oral function and appendicular skeletal muscle mass in elderly persons requiring nursing care $(7,8)$.

(Primary Investigation Items)

Masseter muscle thickness (MMT): This was the primary investigation item of the present study. Based on the method by Ohara et al. (9), we used the ultrasonography device 'Miru-Cube' (Global Health Co., Ltd., Kanagawa, Japan) to perform the measurements. The masseter muscle thickness was measured in a relaxed state. The image display mode was B-mode, and the probe frequency was $6 \mathrm{MHz}$. After palpating the masseter muscle, we placed the probe parallel to the region corresponding to the masseter muscle on a line extending from the corner of the mouth to the mandibular plane and measured the thickness of the masseter muscle twice at rest using the measurement computer screen and calculated the mean score.

Skeletal Muscle Index (SMI): This was the evaluation point of interest of the present study. We used bioelectrical impedance analysis(BIA) to measure skeletal muscle mass. Then we divided the measured muscle mass by the squared height $(\mathrm{m})$, and the adjusted extremity skeletal muscle mass was used as the skeletal muscle index (SMI). InBody ${ }^{\circledR} \mathrm{S} 10$ (InBody Corporation, Seoul, Korea) was used for the measurement.

(Preliminary Investigation Items of the Questionnaire)

Basic attributes: We investigated sex, age, and degree of long-term nursing care.

Medical history: We investigated whether there was a past 
history of cerebrovascular disease, Parkinson's disease and other neurological disease, depression, and diabetes.

Body Mass Index (BMI): This is the index of adult physique that is calculated as body weight divided by height squared. The cut-off value was based on the 1994 criteria of the World Health Organization (WHO) of $18.5 \mathrm{~kg} / \mathrm{m}^{2}$, and subjects with scores less than this value were assigned to the low body weight group (10).

Barthel Index: This is the index of Activities of Daily Living (ADL). The index that assesses the degree of autonomy of 10 items (meals, moving from the wheelchair to the bed, grooming, toilet, bathing, moving, climbing the stairs, dressing, and bowel and bladder control) in several stages (11).

MNA ${ }^{\circledR}-\mathrm{SF}$ (Mini Nutrition Assessment Short Form): This is the index of nutrition status. This is a simple screening method to assess nutritional status in elderly persons aged 65 years or older using six items ('decreased food intake', 'decreased body weight', 'mobility', 'mental stress and acute disease', 'dementia', 'depression', and 'BMI') (12).

$\mathrm{MNA}^{\circledR}$-SF is registered trademarks of Société des Produits Nestlé S.A.

(Items Measured by the Investigators)

Number of present teeth/number of functional teeth: The number of present teeth was set as the number of remaining teeth, excluding residual dental roots, and the number of functional teeth was set as the number of present teeth in addition to the number of prosthetic teeth (e.g., dentures, bridge pontic, and implants).

Use of dentures: We confirmed the use of dentures (total or partial dentures) at time of the investigation.

Clinical Dementia Rating (CDR): Method to assess the severity of dementia. The primary caregivers who sufficiently understood the daily life of the subjects evaluated six items (memory, orientation, judgment and problem solving, social adaptation, and family situation) using a five-stage scale, and based on the results, the researchers (professionals such as physicians or nurses) made an evaluation based on a five-point scale $(0,0.5,1,2$, or 3$)$ (13).

\section{Statistical analysis}

Regarding the primary investigation items and other items, the participants were assigned to two groups (low and high MMT groups) based on the median MMT separated by sex, and intergroup comparisons were performed. Because previous studies did not indicate a clear cut-off value for
MMT (9). We adopted the median men and women scores as the cut-off values in the present study. The MannWhitney $\mathrm{U}$ test was performed to examine continuous variables between the groups and chi-square test was conducted to examine categorical variables.

Based on the results of intergroup comparison, we performed binary logistic regression analysis with stepwise method (variable elimination method) in order to extract the low and high scores of MMT as objective variables and factors influencing them. The selection criteria for independent variables were a significant probability of less than 0.1 and a correlation coefficient less than 0.8 in the simple comparison of high-MMT group and low-MMT group. Because age and sex were adjustment factors, they were included regardless of the significance of probability in the simple comparison. SPSS Statistics 20.0 (IBM Corporation, USA) was used for statistical analysis, and statistical significance was set at $5 \%$.

\section{Ethical considerations}

The present study was approved by the institutional review boards of Tokyo Metropolitan Institute of Gerontology (approval number: 23-1253) and Nihon University School of Dentistry at Matsudo (approval number: EC14-027), and consent was obtained in writing from all subjects and their family members or primary caregiver after receiving individual explanations in writing.

\section{Results}

\section{Basic attributes (Table 1)}

The number of men and women participants in the present study was as follows: 60 men $(21.8 \%)$ and 215 women (78.2\%). The mean ages of men and women were $83.9 \pm 8.0$ and $86.1 \pm 6.0$ years, respectively. SMI (men: 5.8 \pm 1 .3, women: $4.3 \pm 1.2, P<0.001$ ), number of present teeth (men: $5.2 \pm 7.5$ teeth, women: $3.1 \pm 5.8$ teeth, $P=0.040$ ), MNA ${ }^{\circledR}$-SF score (men: $10.4 \pm 2.6$ points, women: $9.3 \pm 2.6$ points, $P=0.004$ ), and Cerebrovascular disease (men: 56.7 \%, women: $29.8 \%, P<0.001)$ revealed significantly lower scores in the women in comparison with the men. The median MMT of men and women were $10.1 \mathrm{~mm}$ and 9.5 $\mathrm{mm}$. Thereafter, participants were assigned to two groups based on the median MMT.

Comparison of high-MMT and low-MMT groups (Table 2)

First, the low and high MMT groups comprised 132 
Table 1. Participants characteristics and sex differences

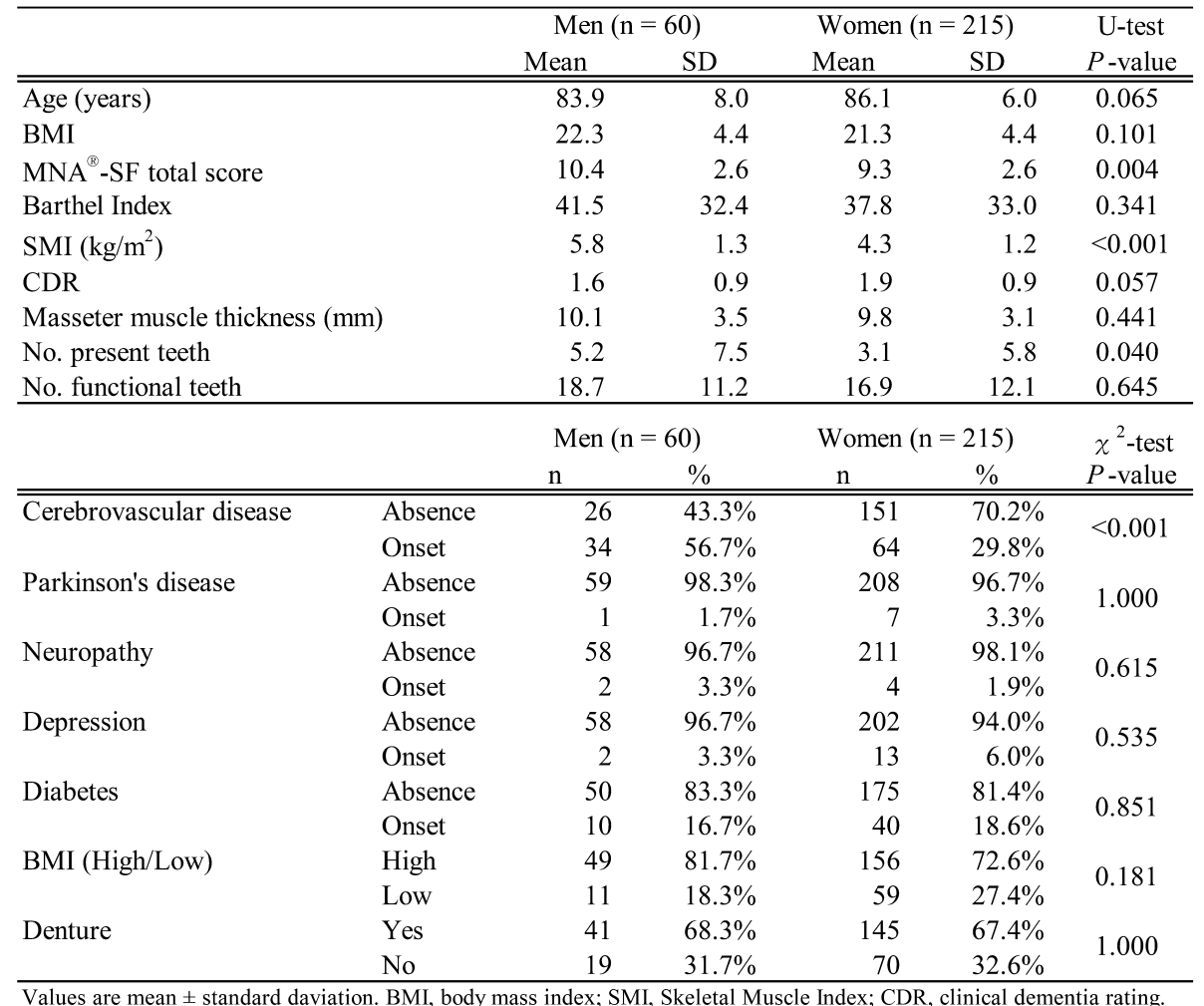

participants (48.0\%) and 143 participants (52.0\%), respectively. The mean SMI score in the high and low-MMT groups was $4.8 \pm 1.4 \mathrm{~kg} / \mathrm{m}^{2}$ and $4.4 \pm 1.4 \mathrm{~kg} / \mathrm{m}^{2}$, respectively, and a significantly higher score was noted in the high-MMT group compared with the low-MMT group $(P=$ 0.010). Furthermore, BMI (high-MMT group: $22.6 \pm 4.6$, low-MMT group: $20.3 \pm 4.0, P<0.001)$, number of functional teeth (high-MMT group: $19.0 \pm 11.4$ teeth, low-MMT group: $15.4 \pm 12.2$ teeth, $P=0.020$ ), Barthel Index (highMMT group: $43.1 \pm 32.5$ points, low-MMT group: $33.8 \pm$ 32.6 points, $P=0.017$ ), and total MNA ${ }^{\circledR}$-SF score (highMMT group: $10.0 \pm 2.7$ points, low-MMT group: $9.1 \pm 2.5$ points, $P=0.003$ ) revealed significantly higher scores in the high-MMT group in comparison with the low-MMT group. Compared with the high-MMT group, CDR was significantly higher in the low-MMT group (high-MMT group: 1.7 \pm 1.0 , low-MMT group $2.0 \pm 0.9$ ). Examination of categorical variables revealed a higher BMI score in the high-MMT group compared with the low-MMT group $(P=0.026)$. In addition, although insignificant, age (high-MMT group: 85.1 \pm 6.6 years, low-MMT group: $86.2 \pm 6.4$ years, $P=0.152$ ) tended to be higher in the low-MMT group compared with the high-MMT group.
Examination of factors related to masseter muscle thickness (Table 3)

As a result of binary logistic regression analysis using the stepwise method, we extracted SMI as a significant factor related to $\operatorname{MMT}(\mathrm{OR}=0.83,95 \% \mathrm{CI}=0.69-0.99, P=0.049)$. Furthermore, although the number of functional teeth $(\mathrm{OR}=$ $0.98,95 \% \mathrm{CI}=0.96-1.00, P=0.065)$ was also not significant, we extracted the items that had the best fit in the final step.

\section{Discussion}

In the present study, we elucidated the relationship between MMT and SMI, with the aim of examining the specific relationship between decreased masticatory function and sarcopenia. Therefore, we conducted a crosssectional study targeting NC. The results revealed a relationship between MMT and SMI. Previous studies have indicated a relationship between masticatory function and sarcopenia in healthy elderly persons (6) in addition to a relationship between swallowing function and SMI in NC (7). To the best of our knowledge, there are no studies that examined the relationship between masticatory function and related factors in NC. Thus, it is our opinion that the findings in the present study are novel. Because it has been 
Table 2. Comparison of high and low MMT group

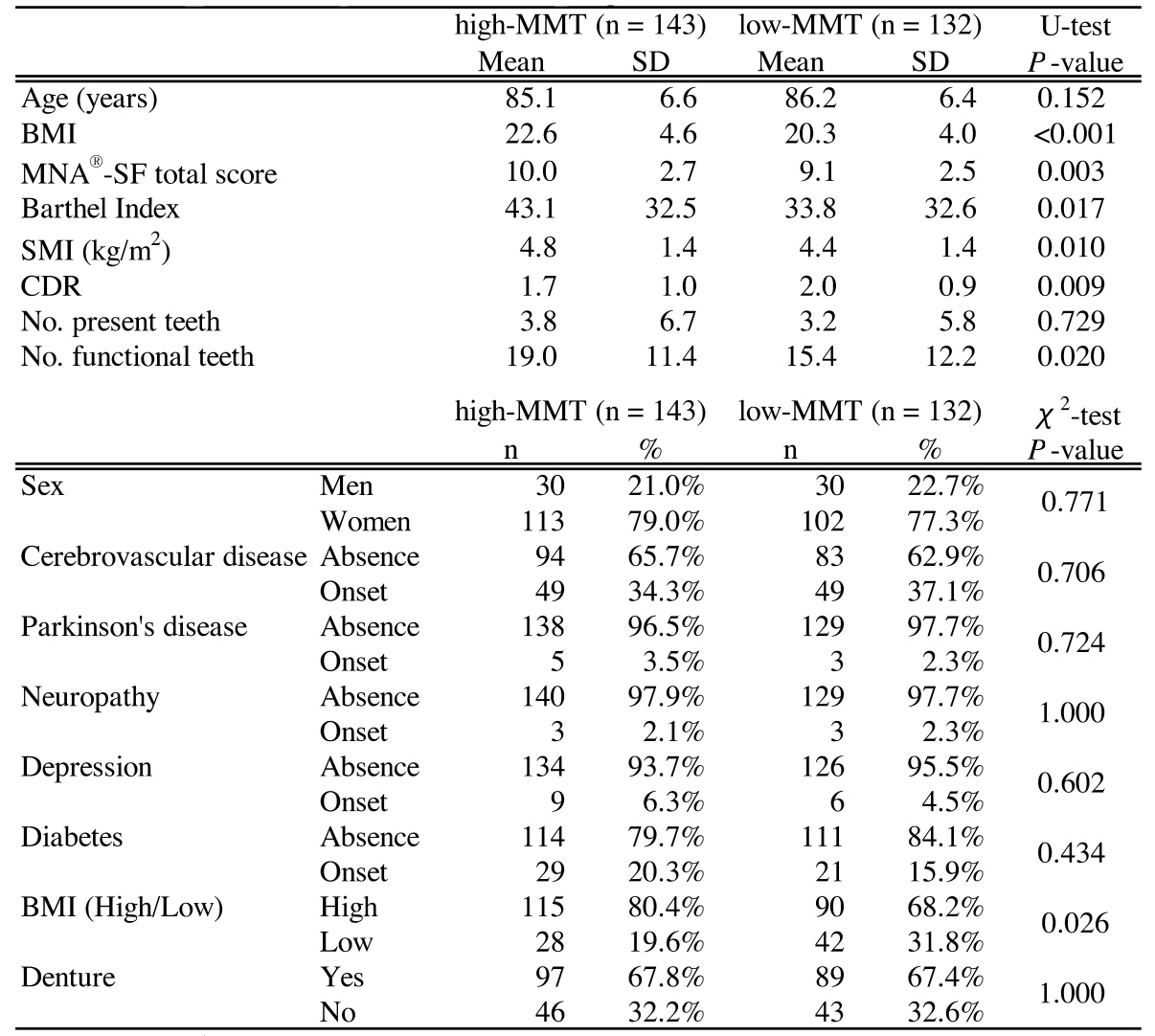

Values are mean \pm standard daviation. MMT, masseter muscle thickness; BMI, body mass index; SMI, Skeletal Muscle Index; CDR, clinical dementia rating.

Table 3. Examination of between various items and masseter muscle thickness

\begin{tabular}{|c|c|c|c|c|c|c|c|}
\hline \multirow[b]{2}{*}{ Variable } & \multirow[b]{2}{*}{ Cutoff } & \multicolumn{3}{|c|}{ Step 1} & \multicolumn{3}{|c|}{ Step 6} \\
\hline & & OR & $95 \% \mathrm{CI}$ & $P$-value & OR & $95 \% \mathrm{CI}$ & $P$-value \\
\hline Sex & 0:Men 1:Women & 0.57 & $0.29-1.15$ & 0.117 & & & \\
\hline Age & & 1.02 & $0.98-1.06$ & 0.356 & & & \\
\hline SMI & & 0.82 & $0.64-1.07$ & 0.147 & 0.83 & $0.69-0.99$ & 0.049 \\
\hline Functional teeth & & 0.98 & $0.96-1.01$ & 0.192 & 0.98 & $0.96-1.00$ & 0.065 \\
\hline Barthel Index & & 1.00 & $0.99-1.02$ & 0.597 & & & \\
\hline MNA ${ }^{\circledR}$-SF total score & & 0.95 & 0.84-1.09 & 0.486 & & & \\
\hline CDR & & 1.12 & $0.77-1.61$ & 0.557 & & & \\
\hline BMI & 0:High 1:Low & 1.18 & $0.60-2.31$ & 0.632 & & & \\
\hline
\end{tabular}

shown that the number of $\mathrm{NC}$ who have impairment of masticatory function of the tongue, etc., is increasing regardless of the maintenance of number of present teeth (3), the results of our study may provide a useful hint in elucidating the cause of impairment.

The SMI, which was examined in the present study, is widely utilized around the world as the diagnostic criterion for sarcopenia; measurement using the BIA method is adopted by the Asian Sarcopenia Consensus (14). However, while the masseter muscle is the representative masticatory muscle, it is also easily accessible for measurement of thickness using an ultrasonography device from the body surface and is suitable for use in large-scale studies. A relationship between MMT and occlusal strength has been demonstrated in past research (15), and MMT is thought to be an effective indicator for predicting the relationship with masticatory function. Furthermore, the merits of investigating this parameter in $\mathrm{NC}$ are as follows: it represents an 
objective index that is not significantly influenced by the degree of cooperation of subjects, and it can be conducted in elderly persons who have dementia.

At first, as a basic attribute of the participants, men showed significantly higher scores of SMI, MNA-SF, number of present teeth, and cerebrovascular disease than women. Generally it is said that men have more SMI, and this result is considered to represent the universality of the participants.

The results of the present study revealed that SMI was significantly higher in the high-MMT group in comparison with the low-MMT group and SMI was extracted as a related factor for MMT. The relationship between swallowing function and SMI in NC has been previously reported by Murakami et al. (7). Furthermore, decrease in activity, deterioration of nutritional status, increase in inflammatory cytokines, oxidative stress, and reduced growth and sex hormones (e.g., testosterone) have also been reported as factors related to decreased muscle mass (16). That is, it appears that decreased muscle mass observed in NC who have decreased physical functions occurs systemically rather than at local sites. Therefore, it is natural that the decrease in muscle mass also develops in the masseter muscle, which is a skeletal muscle similar to those in the extremities. Conversely, it is possible that estimate the SMI from the MMT in NC.

Although the results of the present study revealed that the number of functional teeth was not statistically significant factors, the results of binary logistic regression analysis using stepwise method suggest a relationship with MMT. In previous study by Bhoyar et al., concerning edentulous participants, it was reported that prosthetic treatment was effective in inducing recovery of MMT (17). The number of present teeth of participants in the present study was small (mean number: 3.5 teeth), and 67.6\% (186 participants) wore dentures, because many participants used a prosthetic device such as dentures or bridge. These results suggest that maintenance and recovery of occlusion through the use of prosthetics may be useful in preventing the weakening of the masseter muscle.

$\mathrm{MNA}^{\circledR}-\mathrm{SF}$, which is used to evaluate the risk of poor nutritional status, was not extracted as a related factor by binary logistic regression analysis; however, the results of simple comparison revealed that in comparison with the low-MMT group, the score in the high-MMT group was significantly higher. A study concerning Japanese NC reported that poor nutritional status is a risk factor for sarcopenia in NC because the score was significantly lower in the sarcopenia group compared with the non-sarcopenia group (18). The results of the present study support these previous findings. Also, CDR and BI were not extracted as a related factor. However, the results of simple comparison revealed that in comparison with the low-MMT group, the score in the high-MMT group was significantly higher. Takagi et al. reported that Alzheimer's disease is a risk factor for decreasing SMI (8). Therefore, it is possible that CDR has some influence on masseter muscle.

In the present study, the number of present teeth, which was previously shown to be a factor related to MMT(19), was not extracted as a related factor. Muscle strength generated by the masticatory muscles is finally output as occlusal force through the jawbone and teeth; however, many previous studies examined the relationship between masticatory function and the masseter muscle in younger subjects in whom the number of present teeth was maintained to a certain extent. In contrast, the small mean number of present teeth in participants in the present study and the use of prosthetic devices may have influenced the relationship with the number of present teeth.

A relationship between poor nutritional status and mortality risk has been reported in NC (20). Based on the results of the present study, sarcopenia affects the masticatory muscles, and due to a reduced number of present teeth, masticatory function decreases, and a poor nutritional status develops as a result. Therefore, increased exacerbation of sarcopenia and mortality are possible risks in this elderly group. As mentioned previously, it is possible that the use of a prosthetic device such as dentures may prevent the weakening of the masseter muscle; however, since the use of dentures is difficult in $\mathrm{NC}$ due to decreased physical ability, dementia, etc., such persons are unable to use dentures (21). By contrast, in an interventional study involving elderly persons residing in facilities, Kanehisa et al. reported that the use of dentures is effective in ameliorating nutritional status (22). When compared with the results of the present study, it may be possible to prevent weakening of the masseter muscle in $\mathrm{NC}$ by maintaining the number of functional teeth through the use of prosthetics. As a result of maintenance of masticatory function, poor nutritional status and aggravation of sarcopenia may be ameliorated. Also, this result indicate the prevention of sarcopenia is may be an important factor for the maintenance of masticatory 
function.

Several limitations of the present study should be mentioned. First, because the present study was a crosssectional survey, it could not elucidate a specific causal relationship between decreased MMT and decreased appendicular skeletal muscle mass. For this reason, in order to elucidate a specific causal relationship, it is necessary to conduct a long-term longitudinal study. Secondary, measurement of MMT was performed by multiple investigators who received prior training; however, the possibility of interrater error cannot be completely excluded. Many of the participants used a prosthetic device such as dentures; however, the fit of the dentures was not considered. Moreover, NC have various background factors such as systemic disease and decreased cognitive function, so a further study that takes into account other factors such as medication use and long-term care status is needed. In the future, we plan to address these issues by conducting a longitudinal study. Nevertheless, the present study is complete enumeration, and the significance is large.

\section{Conclusion}

In conclusion, among NC, SMI was significantly higher in the group with high MMT compared with the low-MMT group. Furthermore, decreased SMI and decreased number of functional teeth were extracted as related factors of decreased MMT, there is a possibility that decreasing muscle mass arise from sarcopenia also develops in the masticatory muscles in NC.

\section{Acknowledgments}

We wish to thank everyone who participated in this research, particularly Dr. Ayako Edahiro, Dr. Yuki Ohara, Dr. Masaharu Murakami, Dr. Yoshiko Motohashi, Dr. Shiho Morishita, Dr. Daisuke Takagi, Dr. Masayasu Ito, and Prof. Yasuhiko Kawai who guided us in our research. This project was supported by a Health and Labor Sciences Research Grant (H25-Choju-Ippan-005).

\section{References}

1. Kikutani T, Yoshida M, Enoki H, Yamashita Y, Akifusa S, Shimazaki Y, Hirano H, Tamura F: Relationship between nutrition status and dental occlusion in community-dwelling frail elderly people. Geriatr Gerontol Int, 13: 50-54, 2013.

2. Iijima K: Upstream preventive strategy for age-related sarcopenia in the elderly: Why do the elderly fall into inadequate nutrition? Ann Jpn Prosthodont Soc, 7: 92-101, 2015. (in Japanese)

3. Kikutani T, Tamura F, Nishiwaki K, Kodama M, Suda M, Fukui T, Takahashi N, Yoshida M, Akagawa Y, Kimura M: Oral motor function and masticatory performance in the community-dwelling elderly. Odontology, 97: 38-42, 2009.

4. Cruz-Jentoft AJ, Baeyens JP, Bauer JM, Boirie Y, Cederholm T, Landi F, Martin FC, Michel JP, Rolland Y, Schneider SM, Topinková E, Vandewoude M, Zamboni M, European Working Group on Sarcopenia in Older People: Sarcopenia: European consensus on definition and diagnosis: Report of the European Working Group on Sarcopenia in Older People. Age Ageing, 39: 412-423, 2010.

5. Vetrano DL, Landi F, Volpato S, Corsonello A, Meloni E, Bernabei R, Onder G: Association of sarcopenia with shortand long-term mortality in older adults admitted to acute care wards: results from the CRIME study. J Gerontol A Biol Sci Med Sci, 69: 1154-1161, 2014.

6. Murakami M, Hirano H, Watanabe Y, Sakai K, Kim H, Katakura A: Relationship between chewing ability and sarcopenia in Japanese community-dwelling older adults. Geriatr Gerontol Int, 15: 1007-1012, 2014.

7. Murakami K, Hirano H, Watanabe Y, Edahiro A, Ohara Y, Yoshida H, Kim H, Takagi D, Hironaka S: Relationship between swallowing function and the skeletal muscle mass of older adults requiring long-term care. Geriatr Gerontol Int, 15: 1185-1192, 2015.

8. Takagi D, Hirano H, Watanabe Y, Edahiro A, Ohara Y, Yoshida H, Kim H, Murakami K, Hironaka S: Relationship between skeletal muscle mass and swallowing function in patients with Alzheimer's disease. Geriatr Gerontol Int, doi: 10.1111/ggi.12728, 2016. (in press)

9. Ohara Y, Hirano H, Watanabe Y, Edahiro A, Sato E, Shinkai S, Yoshida H, Mataki S: Masseter muscle tension and chewing ability in older persons. Geriatr Gerontol Int, 13: 372-377, 2013.

10. Woo J, Chi I, Hui E, Chan F, Sham A: Low staffing level is associated with malnutrition in long-term residential care homes. Eur J Clin Nutr, 59: 474-479, 2015.

11. Mahoney FI, Barthel DW: Functional evaluation: the Barthel index. Md State Med J, 14: 61-65, 1965.

12. Kuzuya M, Kanda S, Koike T, Suzuki Y, Satake S, Iguchi A: Evaluation of Mini-Nutritional Assessment for Japanese frail elderly. Nutrition, 21: 498-503, 2005.

13. Morris JC: The Clinical Dementia Rating (CDR): Current version and scoring rules. Neurology, 43: 2412-2414, 1993.

14. Chen LK, Liu LK, Woo J, Assantachai P, Auyeung TW, Bahyah KS, Chou MY, Chen LY, Hsu PS, Krairit O, Lee JS, Lee WJ, Lee Y, Liang CK, Limpawattana P, Lin CS, Peng LN, Satake S, Suzuki T, Won CW, Wu CH, Wu SN, Zhang T, Zeng P, Akishita M, Arai H: Sarcopenia in Asia: consensus report of 
the Asian Working Group for Sarcopenia. J Am Med Dir Assoc, 15: 95-101, 2014.

15. Bakke M, Tuxen A, Vilmann P, Jensen BR, Vilmann A, Toft $\mathrm{M}$ : Ultrasound image of human masseter muscle related to bite force, electromyography, facial morphology, and occlusal factors. Scand J Dent Res, 100: 164-171, 1992.

16. Kamel HK: Sarcopenia and aging. Nutr Rev, 61: 157-167, 2003.

17. Bhoyar PS, Godbole SR, Thombare RU, Pakhan AJ: Effect of complete edentulism on masseter muscle thickness and changes after complete denture rehabilitation: an ultrasonographic study. J Investig Clin Dent, 3: 45-50, 2012.

18. Kamo T, Suzuki R, Ito K, Sugimoto T, Murakoshi T, Nishida Y: Prevalence of Sarcopenia and Its Relation to Body Composition, Physiological Function, and Nutritional Status in Community-dwelling Frail Elderly People, Physical Therapy Japan, 40: 414-420, 2013. (in Japanese)

19. Tetsuka M, Saga T, Nakamura M, Tabira Y, Kusukawa J,
Yamaki K: Relationship between Masseter Muscle Form and Occlusal Supports of Remaining Teeth. Kurume Med J, 59: 5-15, 2012.

20. Lilamand M, Kelaiditi E, Demougeot L, Rolland Y, Vellas B, Cesari M: The Mini Nutritional Assessment-Short Form and mortality in nursing home residents-results from the INCUR study. J Nutr Health Aging, 19: 383-388, 2015.

21. Minakuchi S, Takaoka S, Ito J, Kokubu K, Miyashita K, Shimoyama K, Uematsu H: Diet type and situation of denture wearing in institutionalized elderly and associated factors, Ronen Shika Igaku, 20: 180-186, 2005-2006. (in Japanese)

22. Kanehisa Y, Yoshida M, Taji T, Akagawa Y, Nakamura H: Body weight and serum albumin change after prosthodontic treatment among institutionalized elderly in a long-term care geriatric hospital. Community Dent Oral Epidemiol, 37: 534-538, 2009. 